Piotr Knosalla (Opole)

\title{
GLOBAL SOLUTIONS OF AEROTAXIS EQUATIONS
}

Abstract. We study the existence of the global solutions in a model describing the evolution of density of bacteria and oxygen dissolved in water filling a capillary. In the proof of local existence of classical solutions we use Amann theory. The Moser-Alikakos technique is the main tool for the proof of $L^{\infty}$ boundedness of local solutions.

1. Introduction. Aerotaxis is the movement of bacteria toward the optimal concentration of oxygen for their growth [T], [B], G], MZM], KN]. In $[\mathrm{KN}]$ we introduced a model of aerotaxis which is a modification of those in [TCD], W].

We denote by $u(x, t)$ the density of the colony of bacteria living in a capillary of unit length and by $p(x, t)$ the density of oxygen, for $x \in[0,1]$ and $t>0$.

The evolution of $u(x, t)$ is given by the following one-dimensional driftdiffusion equation:

$$
u_{t}=u_{x x}-\left(u(E(p))_{x}\right)_{x} .
$$

Here $E(p)$ is the energy function, which has a maximum at a point $p^{*}$ of the optimal concentration of oxygen.

We make the following assumptions on the function $E$ :

1. $E$ is a nonnegative $C^{3}$ function on $\mathbb{R}$ with bounded first derivative,

2. $E(p) \equiv 0$ for $p \leq 0$ and $E(p)>0$ for $p>0$,

3. $E$ has only one local maximum at a point $p^{*}$,

4. $\lim _{p \rightarrow \infty} E(p)=0$.

2010 Mathematics Subject Classification: Primary 35B45; Secondary 92C45.

Key words and phrases: aerotaxis, existence of solutions, classical solutions, global solutions.

Received 31 March 2016; revised 13 December 2016.

Published online 4 May 2017. 
The diffusion term $u_{x x}$ is responsible for random walk of bacteria, and the drift term $\left(u(E(p))_{x}\right)_{x}$ for their tendency to achieve the optimal concentration of oxygen. The assumptions on the function $E$ and the form of the drift term describe the impact of oxygen concentration on bacteria movement, namely bacteria escape from regions with too high (too low respectively) concentrations of oxygen and move to the optimal one.

Oxygen diffuses in water filling the capillary and is consumed by the cells of bacteria at the rate proportional to the density of bacteria and the value of the energy function at a given density of oxygen. Thus the evolution of the oxygen density $p(x, t)$ is described by the equation

$$
p_{t}=p_{x x}-E(p) u .
$$

The density $u(x, t)$ satisfies the no-flux boundary condition

$$
u_{x}(0, t)-u(0, t)(E(p))_{x}(0, t)=u_{x}(1, t)-u(1, t)(E(p))_{x}(1, t)=0,
$$

which guarantees that the total mass of bacteria is conserved (see Lemma 2.1). The left end of the capillary is closed. Hence at $x=0$ we impose the no-flux boundary condition

$$
p_{x}(0, t)=0 .
$$

At $x=1$ we may consider two distinct boundary conditions, either a constant level of oxygen at the right end of the capillary:

the Dirichlet condition $p(1, t)=\bar{p}$,

or a constant flow of oxygen across the right end of the capillary:

$$
\text { the Neumann condition } p_{x}(1, t)=\bar{p} \text {. }
$$

Here $\bar{p}$ is a given positive constant.

Equations (1.1), (1.2) are supplemented with the initial density of bacteria

$$
u(x, 0)=u_{0}(x)
$$

and the initial density of oxygen

$$
p(x, 0)=p_{0}(x) .
$$

Here $u_{0}(x), p_{0}(x)$ are given continuous positive functions on $[0,1]$.

Equations (1.1), (1.2) together with the boundary data $1.3-(1.5), 1.6$ and initial data (1.7), (1.8) describe the evolution of the density of bacteria and of oxygen.

The system (1.1)-(1.8) looks like the classical Keller-Segel model of chemotaxis, in which the existence (in the two-dimensional case) of global solutions depends on one parameter, the total mass of the population of 
microorganisms [Bi, NSY]. In the Keller-Segel model with nonlinear diffusion, even in one space dimension, there exists a solution which blows up in finite time (see [CL]). In our case such phenomena do not occur, i.e. for each initial data global solutions exist. From this point of view our model is similar to the system of equations describing chemotaxis with consumption of chemoatractant [Tao, TW], where, as in our case, global solutions exist.

REMARK 1.1. In [TW the authors considered a similar model with $E(p)=p$ and homogeneous Neumann boundary conditions. As their model has a Lyapunov functional, they were able to prove global existence of classical solutions in the two-dimensional case. It seems that the method used in [TW] does not work in our case. (The main difficulty seems to be that the function $E$ is not monotone.)

Local existence of solutions to our problem is obtained by Amann theory Am1 (Theorem 2.3). The main part of this paper is devoted to $L^{\infty}$ estimates of solutions. We use the Moser-Alikakos technique [Al1, Al2, as in [NSY], Tao].

In the proofs we use estimates which are valid only in the one-dimensional case (see Lemma 3.3). In higher dimensions our system can lead to blow-up phenomena as in the classical Keller-Segel system.

The existence, nonexistence and uniqueness of stationary solutions of our system was investigated in $[\mathrm{KN}]$. We proved that the existence and uniqueness of stationary solutions depends on relations between the total mass of the colony of bacteria and on the maximum of the energy function.

2. Local existence. We begin the analysis by proving some a priori properties of classical solutions of our problem.

Lemma 2.1. Let $(u(x, t), p(x, t))$ be the classical solution of the Dirichlet or Neumann problem on an interval $[0, T]$. Then for $t \in[0, T]$,

$$
\begin{aligned}
& u(x, t) \geq 0, \quad p(x, t) \geq 0, \\
& \int_{0}^{1} u_{0}(x) d x=\int_{0}^{1} u(x, t) d x .
\end{aligned}
$$

Proof. By the assumption that $u_{0}(x)>0$ and from the continuity of $u(x, t)$ there exists $\delta \in(0, T)$ such that $u(x, t)>0$ for $t \in[0, \delta]$. The solution $u(x, t)$ fulfills the equation

$$
u_{t}-u_{x x}+E(p)_{x} u_{x}+E(p)_{x x} u=0
$$

under the no-flux boundary condition (1.3). All the coefficients in (2.3) are bounded on $[0,1] \times[\delta, T]$. The maximum principle for parabolic equations (see [PW], [LSU] $)$ gives $u(x, t) \geq 0$ for $(x, t) \in[0,1] \times[\delta, T]$. 
Integrating (1.1) over $[0,1]$ and using the boundary condition (1.3) we obtain the conservation of mass $(2.2)$.

Nonnegativity of $p(x, t)$ for $t \in[0, T]$ follows from the assumption that $E(p) \equiv 0$ for $p \leq 0$ and the maximum principle.

The next lemma gives some estimates on the function $p$.

Lemma 2.2. Let $p(x, t)$ be a classical solution of 11.2 on $[0, T)$. Then for the Dirichlet problem (1.2), 1.4), (1.5) we have

$$
\sup _{t \in[0, T)}|p(\cdot, t)| \leq \max \left\{\bar{p}, \sup _{x \in[0,1]} p_{0}(x)\right\} .
$$

A solution $p(x, t)$ of the Neumann problem (1.2), (1.4), (1.6) is a subsolution of the linear problem

$$
\left\{\begin{array}{l}
v_{t}=v_{x x} \\
v_{x}(0, t)=0, \quad v_{x}(1, t)=\bar{p}, \\
v(x, 0)=v_{0}(x) \geq 0,
\end{array}\right.
$$

i.e.

$$
p(x, t) \leq v(x, t) \quad \text { for } x \in[0,1], t \in[0, T) .
$$

Proof. Inequality (2.4) is an easy consequence of the maximum principle for parabolic equations $[\mathrm{PW}$. To prove $(2.5)$ we introduce the auxiliary function $w(x, t)=v(x, t)-p(x, t)$. Note that $w$ is a solution of the parabolic problem

$$
\left\{\begin{array}{l}
w_{t}=w_{x x}+E(p) u, \\
w_{x}(0, t)=0, w_{x}(1, t)=0, \\
w(x, 0)=0 .
\end{array}\right.
$$

Now we can apply the maximum principle for $w$ to obtain $w(x, t) \geq 0$.

For our purposes we reformulate problems $(1.1)-(1.5)$ and $(1.1)-(1.4)$, (1.6) by introducing a new function $Q$.

For the Dirichlet problem we define $Q(x, t):=p(x, t)-\bar{p}$. Then system (1.1) - 1.5 reads

$$
\left\{\begin{array}{l}
u_{t}=\left(u_{x}-u E(Q+\bar{p})_{x}\right)_{x} \\
Q_{t}=Q_{x x}-E(Q+\bar{p}) u \\
u_{x}(x, t)-\left.u(x, t) E(Q(x, t)+\bar{p})_{x}\right|_{x=0,1}=0 \\
Q_{x}(0, t)=Q(1, t)=0 \\
u(x, 0)=u_{0}(x), \quad Q(x, 0)=Q_{0}(x)=p_{0}(x)-\bar{p}
\end{array}\right.
$$

For the Neumann problem (1.1)-(1.4), (1.6) we introduce the new function 
$Q(x, t)=p(x, t)-\bar{p} x^{2} / 2$ and obtain the following system for $u$ and $Q:$

$$
\left\{\begin{array}{l}
u_{t}=\left(u_{x}-u E\left(Q+\bar{p} x^{2} / 2\right)_{x}\right)_{x}, \\
Q_{t}=Q_{x x}-E\left(Q+\bar{p} x^{2} / 2\right) u+\bar{p}, \\
u_{x}(x, t)-\left.u(x, t) E\left(Q(x, t)+\bar{p} x^{2} / 2\right)_{x}\right|_{x=0,1}=0, \\
Q_{x}(0, t)=Q_{x}(1, t)=0, \\
u(x, 0)=u_{0}(x), \quad Q(x, 0)=Q_{0}(x)=p_{0}(x)-\bar{p} x^{2} / 2 .
\end{array}\right.
$$

The following statement is a consequence of Amann theory [Am1].

Theorem 2.3. Let $\left(u_{0}, Q_{0}\right) \in\left(W^{1, r}(0,1)\right)^{2}$ for some $r>1$. Then systems (2.7) and 2.8 each have a unique local in time classical solution

$$
(u, Q) \in\left(C\left(\left[0, T_{m}\right) \times[0,1]\right) \cap C^{1,2}\left(\left(0, T_{m}\right) \times[0,1]\right)\right)^{2},
$$

where $T_{m}$ denotes the maximal time of existence of solution. Moreover we have the continuation principle: if additionally for each $T>0$ there exists a constant $C(T)$ such that

$$
|(u(\cdot, t), Q(\cdot, t))|_{\infty} \leq C(T) \quad \text { for } 0<t<\min \left\{T, T_{m}\right\},
$$

then $T_{m}=\infty$.

Proof. We set $z=(u, Q)$ and denote by $\mathcal{M}^{2}(\mathbb{R})$ the space of $2 \times 2$ real matrices. Next for $\eta=\left(\eta_{1}, \eta_{2}\right) \in \mathbb{R}^{2}$ and $x \in[0,1]$ we define $a_{D}, a_{N_{1}}, a_{N_{2}} \in$ $C^{2}\left(\mathbb{R}^{2} \times[0,1] ; \mathcal{M}^{2}(\mathbb{R})\right)$ by

$$
\begin{aligned}
& a_{D}(\eta)=\left(\begin{array}{cc}
1 & -\eta_{1} E^{\prime}\left(\eta_{2}+\bar{p}\right) \\
0 & 1
\end{array}\right), \quad a_{N_{1}}(\eta)=\left(\begin{array}{cc}
1 & -\eta_{1} E^{\prime}\left(\eta_{2}+\bar{p} x^{2} / 2\right) \\
0 & 1
\end{array}\right), \\
& a_{N_{2}}(\eta)=\left(\begin{array}{cc}
-x \bar{p} E^{\prime}\left(\eta_{2}+\bar{p} x^{2} / 2\right) & 0 \\
0 & 0
\end{array}\right),
\end{aligned}
$$

and $\mathcal{F}_{D}, \mathcal{F}_{N} \in C^{2}\left(\mathbb{R}^{2} \times[0,1], \mathbb{R}^{2}\right)$ by

$$
\mathcal{F}_{D}(\eta)=\left(\begin{array}{c}
0 \\
-E\left(\eta_{2}+\bar{p}\right) \eta_{1}
\end{array}\right), \quad \mathcal{F}_{N}(\eta)=\left(\begin{array}{c}
0 \\
\bar{p}-E\left(\eta_{2}+\bar{p} x^{2} / 2\right) \eta_{1}
\end{array}\right) .
$$

We introduce the operators

$$
\begin{aligned}
\mathcal{A}_{D}(\eta) z & =-\left(a_{D}(\eta) z_{x}\right)_{x}=-\left(a_{D}(\eta)\left(\begin{array}{c}
u_{x} \\
Q_{x}
\end{array}\right)\right)_{x}, \\
\mathcal{B}_{D}(\eta) z & =b a_{D}(\eta) z_{x}+\left(I_{2}-b\right) z \\
& =b\left(a_{D}(\eta)\left(\begin{array}{c}
u_{x} \\
Q_{x}
\end{array}\right)+\left(I_{2}-b\right)\left(\begin{array}{c}
u \\
Q
\end{array}\right)\right)
\end{aligned}
$$


and

$$
\begin{aligned}
\mathcal{A}_{N}(\eta) z & =-\left(a_{N_{1}}(\eta) z_{x}+a_{N_{2}}(\eta) z\right)_{x} \\
& =-\left(a_{N_{1}}(\eta)\left(\begin{array}{c}
u_{x} \\
Q_{x}
\end{array}\right)+a_{N_{2}}(\eta)\left(\begin{array}{l}
u \\
Q
\end{array}\right)\right)_{x} \\
\mathcal{B}_{N}(\eta) z & =I_{2}\left(a_{N_{1}}(\eta) z_{x}+a_{N_{2}}(\eta) z\right) \\
& =I_{2}\left(a_{N_{1}}(\eta)\left(\begin{array}{c}
u_{x} \\
Q_{x}
\end{array}\right)+a_{N_{2}}(\eta)\left(\begin{array}{l}
u \\
Q
\end{array}\right)\right)
\end{aligned}
$$

where

$$
I_{2}=\left(\begin{array}{ll}
1 & 0 \\
0 & 1
\end{array}\right), \quad b=\left(\begin{array}{cc}
1 & 0 \\
0 & \chi(x)
\end{array}\right), \quad \chi(x)= \begin{cases}1 & \text { if } x=0 \\
0 & \text { if } x=1\end{cases}
$$

Then an abstract formulation of (2.7) reads

$$
\begin{aligned}
& z_{t}+\mathcal{A}_{D}(z) z=\mathcal{F}_{D}(z), \\
& \mathcal{B}_{D}(z) z=0, \\
& z_{0}=\left(u_{0}(x), Q_{0}(x)\right) .
\end{aligned}
$$

Similarly, problem 2.8 can be formulated in the following way:

$$
\begin{aligned}
& z_{t}+\mathcal{A}_{N}(z) z=\mathcal{F}_{N}(z), \\
& \mathcal{B}_{N}(z) z=0, \\
& z_{0}=\left(u_{0}(x), Q_{0}(x)\right) .
\end{aligned}
$$

For $\eta \in \mathbb{R}^{2}$ the matrices $a_{D}(\eta), a_{N_{1}}(\eta)$ have 1 as double eigenvalue and the boundary-value problem operators $\left(\mathcal{A}_{D}, \mathcal{B}_{D}\right),\left(\mathcal{A}_{N}, \mathcal{B}_{N}\right)$ are of separated divergence form in the sense of [Am1, Example 4.3(e)]. Thus these operators are normally elliptic in the sense of [Am1, Section 4]. Therefore by Am1, Theorem $14.4 \& 14.6]$ systems 2.7 and $(2.8)$ each have a unique maximal classical solution

$$
(u, Q) \in\left(C\left(\left[0, T_{m}\right) \times[0,1]\right) \cap C^{1,2}\left(\left(0, T_{m}\right) \times[0,1]\right)\right)^{2}
$$

for some $T_{m}>0$.

Theorem 15.5 of Am1] implies the continuation principle.

3. $L^{\infty}$ boundedness. In this section we prove that solutions of (2.7) and of 2.8 obtained in Theorem 2.3 are $L^{\infty}$ bounded. To do so we use the Moser-Alikakos technique introduced in [Al1, Al2]. Let $q \in(1, \infty)$ and define operators $A_{1}$ and $A_{2}$ in $L^{q}(0,1)$ by

$A_{1} f:=-f_{x x}$ with domain $\mathcal{D}\left(A_{1}\right):=\left\{f \in W^{2, q}(0,1): f_{x}(0)=f(1)=0\right\}$, $A_{2} f:=-f_{x x}$ with domain $\mathcal{D}\left(A_{2}\right):=\left\{f \in W^{2, q}(0,1): f_{x}(0)=f_{x}(1)=0\right\}$. 
The operators $A_{1}, A_{2}$ are sectorial in $L^{q}(0,1)$ (see $\underline{\mathrm{Am} 2}$ ). For each $\beta \geq 0$ and $i=1,2$ the fractional power $A_{i}^{\beta}$ of the operator $A_{i}$ is defined, and the domain $X_{i}^{\beta}=\mathcal{D}\left(A_{i}^{\beta}\right)$ with the norm $|f|_{X_{i}^{\beta}}=\left|A_{i}^{\beta} f\right|_{q}$ is a Banach space. We use the following general imbedding result (see [Hen]).

Lemma 3.1. Suppose $\Omega \subset \mathbb{R}^{n}$ is a bounded domain with smooth boundary, $1<q<\infty$, and $A_{i}$ is a sectorial operator in $X=L^{q}(\Omega)$ with $\mathcal{D}\left(A_{i}\right)=X_{i}^{1} \subset W^{2, q}$. Then for $0 \leq \beta \leq 1$, the following continuous inclusions hold:

$$
\begin{array}{ll}
X_{i}^{\beta} \subset W^{k, l}(\Omega) & \text { when } k-n / l<2 \beta-n / q, l \geq q, \\
X_{i}^{\beta} \subset C^{\nu}(\Omega) & \text { when } 0 \leq \nu<2 \beta-n / q .
\end{array}
$$

We denote by $S_{1}(t)$ and $S_{2}(t)$ the analytic semigroups generated by the sectorial operators $-A_{1}$ and $-A_{2}$ respectively.

Lemma 3.2. Let $q_{1} \in[1, \infty), q_{2} \in(1, \infty), q_{1}<q_{2}$, and $\beta \geq 0$. For $i=1,2$ and $t>0$ we have

$$
\begin{aligned}
\left|A_{i}^{\beta} S_{i}(t) f\right|_{q_{2}} & \leq c(\beta) t^{-\beta} e^{-\lambda_{i} t}|f|_{q_{2}}, & & f \in L^{q_{2}}(0,1), \\
\left|S_{i}(t) f\right|_{q_{2}} & \leq c\left(q_{1}, q_{2}\right) t^{-\frac{1}{2}\left(\frac{1}{q_{1}}-\frac{1}{q_{2}}\right)} e^{-\lambda_{i} t}|f|_{q_{1}}, & & f \in L^{q_{1}}(0,1), \\
\left|A_{i}^{\beta} S_{i}(t) f\right|_{q_{2}} & \leq c\left(q_{1}, q_{2}, \beta\right) t^{-\beta-\frac{1}{2}\left(\frac{1}{q_{1}}-\frac{1}{q_{2}}\right)} e^{-\lambda_{i} t}|f|_{q_{1}}, & & f \in L^{q_{1}}(0,1),
\end{aligned}
$$

where $\lambda_{i}>0$ is the first nonzero eigenvalue of $-A_{i}$.

Proof. The first inequality is well known (see [Hen]). The proof of $(3.2)$ can be found in [Am2, Prop. 12.5]. Taking into account (3.1) and (3.2) we obtain (3.3):

$$
\begin{aligned}
\left|A_{i}^{\beta} S_{i}(t) f\right|_{q_{2}} & =\left|A_{i}^{\beta} S_{i}(t / 2) S_{i}(t / 2) f\right|_{q_{2}} \leq c(\beta)(t / 2)^{-\beta} e^{-\lambda_{i} t / 2}\left|S_{i}(t / 2) f\right|_{q_{2}} \\
& \leq c\left(\beta, q_{1}, q_{2}\right) t^{-\beta-\frac{1}{2}\left(\frac{1}{q_{1}}-\frac{1}{q_{2}}\right)} e^{-\lambda_{i} t}|f|_{q_{1}} \text {. }
\end{aligned}
$$

We will need the following lemma.

Lemma 3.3. Let $Q(x, t)$ be a solution of (2.7) (or 2.8)). Then for every $0<\tau<\min \left\{1, T_{m}\right\}$ and $q_{2} \in(1, \infty)$ there exists $c(\tau)>0$ such that

$$
\sup _{t \in\left[\tau, T_{m}\right)}|Q(\cdot, t)|_{1, q_{2}} \leq c(\tau),
$$

in particular

$$
\sup _{t \in\left[\tau, T_{m}\right)}|Q(\cdot, t)|_{1,4} \leq c(\tau) .
$$

Proof. In 2.7) (or (2.8)) $Q(x, t)$ solves the Cauchy problem

$$
\left\{\begin{array}{l}
Q_{t}=-A_{i} Q+g_{i}(x, t) \\
Q_{0}(x)=Q(x, 0)
\end{array} \quad \text { for } i=1,2,\right.
$$


where

$$
\begin{aligned}
g_{1}(x, t) & :=-E(Q(x, t)+\bar{p}) u(x, t), \\
g_{2}(x, t) & :=-E\left(Q(x, t)+x^{2} \bar{p} / 2\right) u(x, t)+\bar{p} .
\end{aligned}
$$

Recalling conservation of mass of $u$ and boundedness of the energy function $E$ we get

$$
\sup _{t \in\left[0, T_{m}\right)}\left|g_{i}(\cdot, t)\right|_{1}<\infty \quad \text { for } i=0,1 .
$$

Using the Duhamel formula, one can rewrite (3.5) in the form

$$
Q(t)=S_{i}(t) Q_{0}+\int_{0}^{t} S_{i}(t-s) g_{i}(s) d s \quad \text { for } t \in\left(0, T_{m}\right) .
$$

Let $\tau<1, q_{2}>1$ and $1 /\left(2 q_{2}\right)+1 / 2>\beta>1 / 2$. By Lemma 3.1 we have

$$
|Q(t)|_{1, q_{2}} \leq\left|A_{i}^{\beta} Q(t)\right|_{q_{2}} \leq\left|A_{i}^{\beta} S_{i}(t) Q_{0}\right|_{q_{2}}+\int_{0}^{t}\left|A_{i}^{\beta} S_{i}(t-s) g_{i}(s)\right|_{q_{2}} d s,
$$

and inequality (3.3) with $q_{1}=1$ gives

$$
|Q(t)|_{1, q_{2}} \leq c t^{-\beta-\frac{1}{2}\left(1-\frac{1}{q_{2}}\right)} e^{-\lambda_{i} t}\left|Q_{0}\right|_{1}+\int_{0}^{t} c(t-s)^{-\beta-\frac{1}{2}\left(1-\frac{1}{q_{2}}\right)} e^{-\lambda_{i} t}\left|g_{i}(s)\right|_{1} d s .
$$

By substitution $\sigma=t-s$ we obtain

$$
|Q(t)|_{1, q_{2}} \leq c t^{-\beta-\frac{1}{2}\left(1-\frac{1}{q_{2}}\right)} e^{-\lambda_{i} t}\left|Q_{0}\right|_{1}+c \int_{0}^{\infty} \sigma^{-\beta-\frac{1}{2}\left(1-\frac{1}{q_{2}}\right)} e^{-\lambda_{i} \sigma} d \sigma .
$$

Finally,

$$
|Q|_{1, q_{2}} \leq c\left(\tau^{-\beta-\frac{1}{2}\left(1-\frac{1}{q_{2}}\right)}+1\right) .
$$

Taking $q_{2}=4$ we get $(3.4)$.

To derive a priori estimates on $u$, we use the following Gagliardo-Nirenberg interpolation inequality [F, Theorem I.10.1]: Let $\Omega \subset \mathbb{R}^{n}$ be a bounded domain with smooth boundary, and let $l, q, r \geq 1$. Then, for any $f \in$ $W^{1, q}(\Omega) \cap L^{r}(\Omega)$, there exist positive constants $c_{1}, c_{2}$ such that

$$
|f|_{l} \leq c_{1}|\nabla f|_{q}^{a}|f|_{r}^{1-a}+c_{2}|f|_{r}
$$

with $a \in[0,1]$ satisfying

$$
\frac{n}{l}=a\left(\frac{n}{q}-1\right)+\frac{n}{r}(1-a) .
$$

We shall also need the Gronwall lemma [E, p. 624]:

LEMma 3.4. Let $y(t)$ be a nonnegative, absolutely continuous function on $[0, T]$ which satisfies for a.e. $t$ the differential inequality

$$
y^{\prime}(t) \leq h(t) y(t)+k(t)
$$


where $h(t)$ and $k(t)$ are nonnegative, integrable functions on $[0, T]$. Then

$$
y(t) \leq \exp \left(\int_{0}^{t} h(s) d s\right)\left(y(0)+\int_{0}^{t} k(s) d s\right) .
$$

Now we are in a position to prove some $L^{s}$ estimates for the solution $u$.

Lemma 3.5. Let $s \geq 2$ and $0<\tau<\min \left\{1, T_{m}\right\}$. There exists $c(\tau, s)$ such that

$$
\sup _{t \in\left[\tau, T_{m}\right)}|u(\cdot, t)|_{s} \leq c(\tau, s),
$$

both for the Neumann and the Dirichlet problem.

Proof. Consider the auxiliary problem

$$
u_{t}=\left(u_{x}-u w_{x}\right)_{x}
$$

with the no-flux boundary conditions

$$
u_{x}(x, t)-\left.u(x, t) w(x, t)_{x}\right|_{x=1,2}=0,
$$

where $w(x, t):=E(Q+\bar{p})$ in the Dirichlet case and $w(x, t):=E\left(Q+\bar{p} x^{2} / 2\right)$ for the Neumann problem. Multiply both sides of $(3.9)$ by $u^{s-1}$ and integrate on $[0,1]$ to get

$$
\begin{aligned}
\frac{1}{s} \frac{d}{d t} \int_{0}^{1} u^{s} d x & =\int_{0}^{1} u^{s-1}\left(u_{x}-u w_{x}\right)_{x} d x \\
& =-(s-1) \int_{0}^{1} u^{s-2} u_{x}\left(u_{x}-u w_{x}\right) d x \\
& =-(s-1) \int_{0}^{1}\left(u^{s / 2-1} u_{x}\right)^{2} d x+(s-1) \int_{0}^{1} u^{s / 2} u^{s / 2-1} u_{x} w_{x} d x \\
& =-\frac{4(s-1)}{s^{2}} \int_{0}^{1}\left(\left(u^{s / 2}\right)_{x}\right)^{2} d x+\frac{2(s-1)}{s} \int_{0}^{1} u^{s / 2}\left(u^{s / 2}\right)_{x} w_{x} d x=: I .
\end{aligned}
$$

By the Young inequality $a b \leq \frac{\varepsilon}{4} a^{2}+\frac{1}{\varepsilon} b^{2}$ we have

$$
\begin{aligned}
I & \leq-\frac{4(s-1)}{s^{2}} \int_{0}^{1}\left(\left(u^{s / 2}\right)_{x}\right)^{2} d x+\frac{2(s-1)}{s} \int_{0}^{1} \frac{s}{4} u^{s}\left(w_{x}\right)^{2}+\frac{1}{s}\left(\left(u^{s / 2}\right)_{x}\right)^{2} d x \\
& =-\frac{2(s-1)}{s^{2}} \int_{0}^{1}\left(\left(u^{s / 2}\right)_{x}\right)^{2} d x+\frac{s-1}{2} \int_{0}^{1} u^{s}\left(w_{x}\right)^{2} d x,
\end{aligned}
$$

so the Schwarz inequality yields

$$
I \leq-\frac{2(s-1)}{s^{2}} \int_{0}^{1}\left(\left(u^{s / 2}\right)_{x}\right)^{2} d x+\frac{s-1}{2}\left(\int_{0}^{1} u^{2 s} d x\right)^{1 / 2}\left(\int_{0}^{1}\left(w_{x}\right)^{4} d x\right)^{1 / 2}
$$


To estimate $\left(\int_{0}^{1} u^{2 s} d x\right)^{1 / 2}$ we use the Gagliardo-Nirenberg inequality 3.7 with $l=4, q=r=2, a=1 / 4$ :

$$
\left(\int_{0}^{1} u^{2 s} d x\right)^{1 / 2}=\left|u^{s / 2}\right|_{4}^{2} \leq c\left(\left|\left(u^{s / 2}\right)_{x}\right|_{2}^{9 / 4}\left|u^{s / 2}\right|_{2}^{11 / 4}+\left|u^{s / 2}\right|_{2}^{2}\right) .
$$

Hence

$$
\begin{aligned}
& \frac{s-1}{2}\left(\int_{0}^{1} u^{2 s} d x\right)^{1 / 2}\left(\int_{0}^{1}\left(w_{x}\right)^{4} d x\right)^{1 / 2}=\frac{s-1}{2}\left|w_{x}\right|_{4}^{2}\left|u^{s / 2}\right|_{4}^{2} \\
& \quad \leq \frac{s-1}{2} c\left(\left|w_{x}\right|_{4}^{2}\left|u^{s / 2}\right|_{2}\left|\left(u^{s / 2}\right)_{x}\right|_{2}^{1 / 2}\left|u^{s / 2}\right|_{2}^{1 / 2}+\left|u^{s / 2}\right|_{2}\left|u^{s / 2}\right|_{2}\left|w_{x}\right|_{4}^{2}\right)=: J .
\end{aligned}
$$

By the Young inequality $a b \leq \frac{\varepsilon}{4} a^{2}+\frac{1}{\varepsilon} b^{2}$ we get

$$
\begin{aligned}
J \leq & \frac{s-1}{2} c\left(\frac{1}{4}\left|w_{x}\right|_{4}^{4}\left|u^{s / 2}\right|_{2}^{2}+\right. \\
+ & \frac{4}{s^{2} c}\left|\left(u^{s / 2}\right)_{x}\right|_{2}^{2} \\
& \left.+\frac{c s^{2}}{16}\left|u^{s / 2}\right|_{2}^{2}+\left|u^{s / 2}\right|_{2}^{2}+\frac{1}{4}\left|u^{s / 2}\right|_{2}^{2}\left|w_{x}\right|_{4}^{4}\right) \\
= & \frac{s-1}{2} c\left|u^{s / 2}\right|_{2}^{2}\left(\frac{1}{2}\left|w_{x}\right|_{4}^{4}+1+\frac{c s^{2}}{16}\right)+\frac{2(s-1)}{s^{2}}\left|\left(u^{s / 2}\right)_{x}\right|_{2}^{2} .
\end{aligned}
$$

Now, by 3.11) and 3.12,

$$
\frac{1}{s} \frac{d}{d t} \int_{0}^{1} u^{s} d x \leq \frac{s-1}{2} c\left|u^{s / 2}\right|_{2}^{2}\left(\frac{1}{2}\left|w_{x}\right|_{4}^{4}+1+\frac{c s^{2}}{16}\right) .
$$

Taking $k(t):=\frac{s(s-1)}{2} c\left(\frac{1}{2}\left|w_{x}\right|_{4}^{4}+1+\frac{c s^{2}}{16}\right), y(t):=\left|u^{s / 2}\right|_{2}^{2}$ in Gronwall's lemma, and recalling (3.4), we obtain the desired estimates.

Lemma 3.6. Let $0<T<\infty$. For system (2.7) (or (2.8)) we have

$$
\sup _{t \in\left[0, T_{m}\right) \cap[0, T]}|u(\cdot, t)|_{\infty}<\infty .
$$

Proof. For the proof we apply the Moser-Alikakos iteration procedure [Al1, Al2]. The idea is to estimate $\sup _{t \in\left[\tau, T_{m}\right)}|u(t)|_{s}$ by $\sup _{t \in\left[\tau, T_{m}\right)}|u(t)|_{s / 2}$. From Lemma 3.5 we have

$$
\sup _{t \in\left[\tau / 2, T_{m}\right)}\left|g_{i}(\cdot, t)\right|_{2}<c_{0}(\tau), \quad i=1,2 .
$$

By the Duhamel formula on $(\tau / 2, t]$ (see Lemma 3.3), for $i=1,2$ we get

$$
Q(t)=S_{i}(t-\tau / 2) Q(\tau / 2)+\int_{\tau / 2}^{t} S_{i}(t-s) g_{i}(s) d s \quad \text { for } t \in\left(\tau / 2, T_{m}\right) .
$$


We pick $\beta \in(3 / 4,1)$. Lemmas 3.1 and 3.3 give

$$
\begin{aligned}
& |Q(\cdot, t)|_{C^{1}[0,1]} \\
& \quad \leq c\left|A_{i}^{\beta} Q(t)\right|_{2} \leq\left|A_{i}^{\beta} S_{i}(t-\tau / 2) Q(\tau / 2)\right|_{2}+\int_{\tau / 2}^{t}\left|A_{i}^{\beta} S_{i}(t-s) g_{i}(s)\right|_{2} d s \\
& \quad \leq c\left(\frac{e^{-\lambda_{i}(t-\tau / 2)}}{(t-\tau / 2)^{\beta}}|Q(\tau / 2)|_{2}+\int_{\tau / 2}^{t} \frac{e^{-\lambda_{i}(t-s)}}{(t-s)^{\beta}}\left|g_{i}(s)\right|_{2} d s\right) \\
& \quad \leq c\left(\frac{e^{-\lambda_{i}(t-\tau / 2)}}{(t-\tau / 2)^{\beta}}|Q(\tau / 2)|_{2}+c_{0}(\tau) \int_{\tau / 2}^{t} \frac{e^{-\lambda_{i}(t-s)}}{(t-s)^{\beta}} d s\right),
\end{aligned}
$$

where $c$ is a constant. It follows that for $w_{x}$ from the auxiliary problem (3.9) -3.10) we have

$$
\sup _{t \in\left[\tau, T_{m}\right)}\left|w(\cdot, t)_{x}\right|_{\infty} \leq c_{1},
$$

where $c_{1}$ depends only on $\tau$. We multiply both sides of $(3.9)$ by $s u^{s-1}$ and integrate on $[0,1]$. We arrive at

$$
\begin{aligned}
\frac{d}{d t} \int_{0}^{1} u^{s} d x & =-\frac{4(s-1)}{s} \int_{0}^{1}\left(\left(u^{s / 2}\right)_{x}\right)^{2} d x+2(s-1) \int_{0}^{1} w_{x} u^{s / 2}\left(u^{s / 2}\right)_{x} d x \\
& \leq-\frac{4(s-1)}{s} \int_{0}^{1}\left(\left(u^{s / 2}\right)_{x}\right)^{2} d x+c_{1}(\tau) 2(s-1) \int_{0}^{1} u^{s / 2}\left|\left(u^{s / 2}\right)_{x}\right| d x .
\end{aligned}
$$

By the Young inequality, the above is

$$
\begin{aligned}
& \leq-\frac{4(s-1)}{s} \int_{0}^{1}\left(\left(u^{s / 2}\right)_{x}\right)^{2} d x+2 c_{1}(s-1)\left(\frac{s c_{1}}{4} \int_{0}^{1} u^{s} d x+\frac{1}{c_{1} s} \int_{0}^{1}\left(\left(u^{s / 2}\right)_{x}\right)^{2} d x\right) \\
& =-\frac{2(s-1)}{s} \int_{0}^{1}\left(\left(u^{s / 2}\right)_{x}\right)^{2} d x+\frac{c_{1}^{2}}{2}(s-1) s \int_{0}^{1} u^{s} d x .
\end{aligned}
$$

Adding $s(s-1) \int_{0}^{1} u^{s} d x$ to both sides we obtain

$$
\begin{aligned}
& \frac{d}{d t} \int_{0}^{1} u^{s} d x+s(s-1) \int_{0}^{1} u^{s} d x \\
& \leq-\frac{2(s-1)}{s} \int_{0}^{1}\left(\left(u^{s / 2}\right)_{x}\right)^{2} d x+c_{2}(s-1) s \int_{0}^{1} u^{s} d x,
\end{aligned}
$$

where $c_{2}=1+c_{1}^{2} / 2$. To estimate the last term in 3.15 we use the GagliardoNirenberg inequality (3.7) with $q=l=2, a=1 / 3, r=1$ :

$$
c_{2}(s-1) s \int_{0}^{1} u^{s} d x=c_{2}(s-1) s\left|u^{s / 2}\right|_{2}^{2} \leq c_{2} c(s-1) s\left(\left|\left(u^{s / 2}\right)_{x}\right|_{2}^{7 / 3}\left|u^{s / 2}\right|_{1}^{8 / 3}+\left|u^{s / 2}\right|_{1}^{2}\right) \text {. }
$$


By the Young inequality $a b \leq \varepsilon a^{3}+\frac{(3 \varepsilon)^{-1 / 2}}{3 / 2} b^{3 / 2}$ with $\varepsilon=\frac{2}{s^{2} c_{2} c}$, the above is

$$
\begin{aligned}
& \leq c_{2} c(s-1) s\left(\frac{2}{s^{2} c_{2} c}\left|\left(u^{s / 2}\right)_{x}\right|_{2}^{2}+\frac{2(3 \varepsilon)^{-1 / 2}}{3}\left|u^{s / 2}\right|_{1}^{2}+\left|u^{s / 2}\right|_{1}^{2}\right) \\
& \leq \frac{2(s-1)}{s}\left|\left(u^{s / 2}\right)_{x}\right|_{2}^{2}+c_{2}(s-1) s c\left(s c_{3}\left|u^{s / 2}\right|_{1}^{2}+\left|u^{s / 2}\right|_{1}^{2}\right)
\end{aligned}
$$

where the constant $c_{3}$ is independent of $s$. For $c_{4}=\max \left\{1, c_{3}\right\}$ we have

$$
c_{2}(s-1) s\left|u^{s / 2}\right|_{2}^{2} \leq \frac{2(s-1)}{s}\left|\left(u^{s / 2}\right)_{x}\right|_{2}^{2}+c_{2} c_{4}(s-1) s c(s+1)\left|u^{s / 2}\right|_{1}^{2} .
$$

Inserting these estimates into 3.15 we get

$$
\frac{d}{d t} \int_{0}^{1} u^{s} d x+(s-1) s \int_{0}^{1} u^{s} d x \leq c_{4}(s-1) s(s+1)\left(\int_{0}^{1} u^{s / 2} d x\right)^{2} .
$$

Hence

$$
\frac{d}{d t}\left[e^{s(s-1)(t-\tau)} \int_{0}^{1} u^{s} d x\right] \leq e^{s(s-1)(t-\tau)} c_{4} s(s-1)(s+1)\left(\int_{0}^{1} u^{s / 2} d x\right)^{2} .
$$

Integrating (3.17) over $[\tau, t]$ for $t \in\left(\tau, T_{m}\right)$ we get

$$
\int_{0}^{1} u^{s} d x \leq \int_{0}^{1} u^{s}(x, \tau) d x+c_{4}(s+1) \sup _{t \in\left[\tau, T_{m}\right]}\left(\int_{0}^{1} u^{s / 2} d x\right)^{2} .
$$

Define

$$
K(s):=\max \left\{|u(\cdot, \tau)|_{\infty}, \sup _{t \in\left[\tau, T_{m}\right]}\left(\int_{0}^{1} u^{s} d x\right)^{1 / s}\right\} .
$$

Then from (3.18) we have

$$
K(s) \leq\left[c_{5}(1+s)\right]^{1 / s} K(s / 2) \quad \text { for all } s \geq 2,
$$

where $c_{5}=c_{4}+1$. Taking $s=2^{j}, j=1,2, \ldots$, we obtain

$$
\begin{aligned}
K\left(2^{j}\right) & \leq c_{5}^{2^{-j}}\left(1+2^{j}\right)^{2^{-j}} K\left(2^{j-1}\right) \\
& \leq c_{5}^{\sum_{i=1}^{j} 2^{-i}}\left(1+2^{j}\right)^{2^{-j}} \cdots(1+2)^{2^{-1}} K(1) \\
& \leq c_{5}\left[2^{j^{-j}}\left(2^{-j}+1\right)^{2^{-j}}\right] \cdots\left[2^{2^{-1}}\left(2^{-1}+1\right)^{2^{-1}}\right] K(1) \\
& \leq c_{5} 2^{\sum_{i=1}^{j} i 2^{-i}} \cdot 2^{\sum_{i=1}^{j} 2^{-i}} K(1) \leq c_{5} 2^{3} K(1) .
\end{aligned}
$$

Letting $j \rightarrow \infty$ yields

$$
\sup _{t \in\left[\tau, T_{m}\right)}|u(\cdot, t)|_{\infty}<c_{5} 2^{3} K(1) .
$$

Since $u \in C\left(\left[0, T_{m}\right) \times[0,1]\right)$ we get $(3.14)$. 
Acknowledgments. The author is grateful to Piotr Biler and Tadeusz Nadzieja for interesting discussions during the preparation of this paper.

\section{References}

[Al1] N. D. Alikakos, An application of the invariance principle to reaction-diffusion equations, J. Differential Equations 33 (1979), 201-225.

[Al2] N. D. Alikakaos, $L^{p}$ bounds of solutions of reaction-diffusion equations, Comm. Partial Differential Equations 4 (1979), 827-868.

[Am1] H. Amann, Nonhomogeneous linear and quasilinear elliptic and parabolic boundary value problems, in: Function Spaces, Differential Operators and Nonlinear Analysis, H. Triebel and H. J. Schmeisser (eds.), Teubner-Texte Math. 133, Teubner, Stuttgart, 1993, 9-126.

[Am2] H. Amann, Dual semigroup and second order linear elliptic boundary value problems, Israel J. Math. 45 (1983), 225-254.

[Bi] P. Biler, Local and global solvability of parabolic systems modelling chemotaxis, Adv. Math. Sci. Appl. 8 (1998), 715-743.

[B] D. Bray, Cell Movements, Garland Sci., 2001.

[CL] T. Cieślak and Ph. Laurençot, Finite time blow-up for a one-dimensional quasilinear parabolic-parabolic chemotaxis system, Ann. Inst. H. Poincaré Anal. Non Linéaire 27 (2010), 437-446.

[E] L. C. Evans, Partial Differential Equations, Amer. Math. Soc., Providence, RI, 1998.

[F] A. Friedman, Partial Differential Equations, Holt, Rinehart \& Winston, New York, 1969.

[G] A. N. Glagolev, Reception of the energy level in bacteria taxis, J. Theor. Biol. 82 (1980), 171-185.

[Hen] D. Henry, Geometric Theory of Semilinear Parabolic Equations, Lecture Notes in Math. 840, Springer, Berlin, 1981.

[KN] P. Knosalla and T. Nadzieja, Stationary solutions of aerotaxis equations, Appl. Math. (Warsaw) 42 (2015), 125-135.

[LSU] O. A. Ladyzhenskaya, V. A. Solonnikov and N. N. Ural'tseva, Linear and Quasilinear Equations of Parabolic Type, Amer. Math. Soc. Transl. 23, Amer. Math. Soc., Providence, RI, 1968.

[MZM] B. C. Mazzag, I. B. Zhulin and A. Mogilner, Model of bacterial band formation in aerotaxis, Biophys. J. 85 (2003), 3558-3574.

[NSY] T. Nagai, T. Senba and K.Yoshida, Application of the Moser-Trudinger inequality to a parabolic system of chemotaxis, Funkcial. Ekvac. 40 (1997), 411-433.

[PW] M. H. Protter and H. F. Weinberger, Maximum Principles in Differential Equations, Springer, 1984.

[Tao] Y. Tao, Boundedness in a chemotaxis model with oxygen consumption by bacteria, J. Math. Anal. Appl. 381 (2011), 521-529.

[TW] Y. Tao and M. Winkler, Eventual smoothness and stabilization of large-data solutions in a three-dimensional chemotaxis system with consumption of chemoattractant, J. Differential Equations 252 (2012), 2520-2543.

[T] B. L. Taylor, I. B. Zhulin and M. S. Johnson, Aerotaxis and other energy-sensing behaviour in bacteria, Ann. Rev. Microbiol. 53 (1999), 103-128. 
[TCD] I. Tuval, L. Cisneros, C. Dombrowski, C. W. Wolgemuth, J. O. Kessler and R. E. Goldstein, Bacterial swimming and oxygen transport near contact lines, Proc. Nat. Acad. Sci. USA 102 (2005), 2277-2282.

[W] M. Winkler, Global large-data solutions in a chemotaxis-(Navier-) Stokes system modeling cellular swimming in fluid drops, Comm. Partial Differential Equations 37 (2012), 319-351.

Piotr Knosalla

Institute of Mathematics and Informatics

Opole University

Oleska 48

45-052 Opole, Poland

E-mail: pknosalla@math.uni.opole.pl 